\title{
Direct Observation of Incoherent Cherenkov Diffraction Radiation in the Visible Range
}

\author{
R. Kieffer, ${ }^{1,{ }^{*}}$ L. Bartnik, ${ }^{3}$ M. Bergamaschi, ${ }^{1}$ V. V. Bleko, ${ }^{2}$ M. Billing, ${ }^{3}$ L. Bobb,${ }^{4}$ J. Conway, ${ }^{3}$ \\ M. Forster, ${ }^{3}$ P. Karataev, ${ }^{5}$ A. S. Konkov, ${ }^{2}$ R. O. Jones, ${ }^{1}$ T. Lefevre, ${ }^{1}$ J. S. Markova, ${ }^{2}$ S. Mazzoni, ${ }^{1}$ \\ Y. Padilla Fuentes, ${ }^{3}$ A. P. Potylitsyn, ${ }^{2}$ J. Shanks, ${ }^{3}$ and S. Wang ${ }^{3}$ \\ ${ }^{1}$ CERN, CH-1211 Geneva 23, Switzerland \\ ${ }^{2}$ Tomsk Polytechnic University, Lenina avenue 30, Tomsk 634050, Russian Federation \\ ${ }^{3}$ Cornell University, Ithaca, New York 14850, USA \\ ${ }^{4}$ Diamond Light Source, Oxfordshire OX11 ODE, United Kingdom \\ ${ }^{5}$ Royal Holloway, University of London, Egham, Surrey TW20 OEX, United Kingdom
}

(Received 3 April 2018; published 1 August 2018)

\begin{abstract}
We report on the observation of incoherent Cherenkov radiation emitted by a $5.3 \mathrm{GeV}$ positron beam circulating in the Cornell electron-positron storage ring as the beam passes in the close vicinity of the surface of a fused silica radiator (i.e., at a distance larger than $0.8 \mathrm{~mm}$ ). The shape of the radiator was designed in order to send the Cherenkov photons towards the detector, consisting of a compact optical system equipped with an intensified camera. The optical system allows both the measurements of $2 \mathrm{D}$ images and angular distribution including polarization study. The corresponding light intensity has been measured as a function of the distance between the beam and the surface of the radiator and has shown a good agreement with theoretical predictions. For highly relativistic particles, a large amount of incoherent radiation is produced in a wide spectral range. A light yield of $0.8 \times 10^{-3}$ photon per particle per turn has been measured at a wavelength of $600 \pm 10 \mathrm{~nm}$ in a $2 \mathrm{~cm}$ long radiator and for an impact parameter of $1 \mathrm{~mm}$. This will find applications in accelerators as noninvasive beam diagnostics for both leptons and hadrons.
\end{abstract}

DOI: 10.1103/PhysRevLett.121.054802

Cherenkov radiation refers to the emission of broadband electromagnetic radiation from a charged particle traveling inside a dielectric at a speed greater than the speed of light in the medium. First observed experimentally by Cherenkov in 1934 [1], a theoretical model, developed by Tamm and Frank [2,3] in 1937, confirmed these initial observations. A quantum theory of uniformly moving electrons inside a medium was then published by Ginzburg in 1940 [4]. Since then, Cherenkov radiation from relativistic charged particles has led to the development of numerous applications in many fields including astrophysics [5] and particle detection and identification $[6,7]$. According to [2], the light intensity scales proportionally to the length of the radiator, giving one the opportunity to produce a large photon flux when using a long radiator. The light is emitted at a characteristic angle $\theta$, defined as $\cos \theta=1 / \beta n$, which depends both on particle velocity $\beta$ and on the index of refraction of the radiator, $n$. This angle is typically large and allows steering the photons

Published by the American Physical Society under the terms of the Creative Commons Attribution 4.0 International license. Further distribution of this work must maintain attribution to the author(s) and the published article's title, journal citation, and DOI. away from the particle beam trajectory, limiting the contamination of the Cherenkov signal by other sources of background light. With such characteristics, Cherenkov radiation has also been regularly used in instrumentation for charged particle accelerators, especially for applications requiring a large flux of photons and a fast time response [8].

The theory of Cherenkov radiation was further developed in 1947 by Ginzburg and Frank [9] who considered the emission of Cherenkov radiation from particles moving parallel to a dielectric boundary. Similar studies were performed by Linhart in 1955 [10] and Ulrich in 1966 [11], establishing that the radiation comes from the interaction between the electromagnetic field of the moving charged particle and the atomic electrons on the surface of the dielectric. The typical size of the electromagnetic field of a particle beam depends on the wavelength $\lambda$ and the beam energy, and typically scales as $\gamma \lambda / 2 \pi$, where $\gamma$ is the Lorentz factor. In this Letter, we refer to Cherenkov diffraction radiation (CDR) as the radiation emitted through the Cherenkov effect by particles traveling at a distance $h$ from the surface of a dielectric, also called the impact parameter.

In 1955, Danos [12] expanded the model of the Cherenkov emission from a single particle radiating incoherent light in the visible range to the coherent emission of a particle beam at wavelengths similar or longer than its 
bunch length. This coherent emission would give rise to high power as it scales with the number of particles squared, contrary to incoherent radiation, which is linearly proportional to the beam current. Experimental validations came later with the development of dielectric Cherenkov masers [13] that produced high output powers of coherent radiation in the microwave regime [14-16] using lowenergy $(<1 \mathrm{MeV})$, very high current $(>1 \mathrm{kA})$ electron beams with pulse durations of tens of nanoseconds. Coherent Cherenkov radiation from short electron bunches was also observed experimentally in 1991 by Ciocci et al. [17] and Ohkuma et al. [18]. Its capability to produce high output powers at millimeter or submillimeter wavelengths has inspired several groups to develop dielectric wakefield acceleration (DWA) [19], that can produce accelerating gradients in excess of $1 \mathrm{GV} / \mathrm{m}$ [20].

Cherenkov diffraction radiation and, more generally, diffraction radiation (DR) [21] can be considered as polarization radiation (PR) resulting from polarization currents occurring in the volume of a dielectric, induced by the electromagnetic field of the passing particles [22-24]. For moderately relativistic particles (i.e., $\gamma$ ranging from 1 to 100) and realistic distances (denoted by the impact parameter) between the dielectric and the beam (i.e., larger than $100 \mu \mathrm{m}$ ), the spectrum of PR would be concentrated in the giga- to terahertz range, as the radiation power reduces exponentially with the photon energy. The spectrum of PR will expand to the near infrared or visible range, for higher particle energies (i.e., $\gamma>10^{3}$ ) even when using impact parameters larger than a millimeter. In this context, incoherent DR has been studied intensively for the last 10 years and has led to the development of noninterceptive beam size [25] and position [26] monitors by measuring photons emitted from thin slits in the visible range. DR is generated by a charge moving in the vicinity of an interface between two media with different permittivity and is typically emitted both in the forward (i.e., along the particle trajectory) and the backward (i.e., along the specular reflection from the interface) directions. Contrary to DR, CDR has the advantage of producing photons not just at the interface but all along the longitudinal surface of the radiator (i.e., parallel to the charged particle beam trajectory), resulting in a significantly higher light intensity. The increase in photon yield is due to the proportional increase of the number of atoms participating in the emission process along the length of the dielectric. Moreover, the Cherenkov photons are emitted at a large angle relative to the particle beam trajectory such that the signal is separated from synchrotron radiation, which is, in the case of DR, the main source of background $[25,26]$.

Experiments were performed on the Cornell Electron Storage Ring (CESR) [27], where a $5.3 \mathrm{GeV}$ positron bunch, with a charge of up to $2.5 \mathrm{nC}$ (equivalent to a circulating beam current of $1 \mathrm{~mA}$ ), produces incoherent $\mathrm{CDR}$ in a $2 \mathrm{~cm}$ long fused silica radiator. Two radiators with

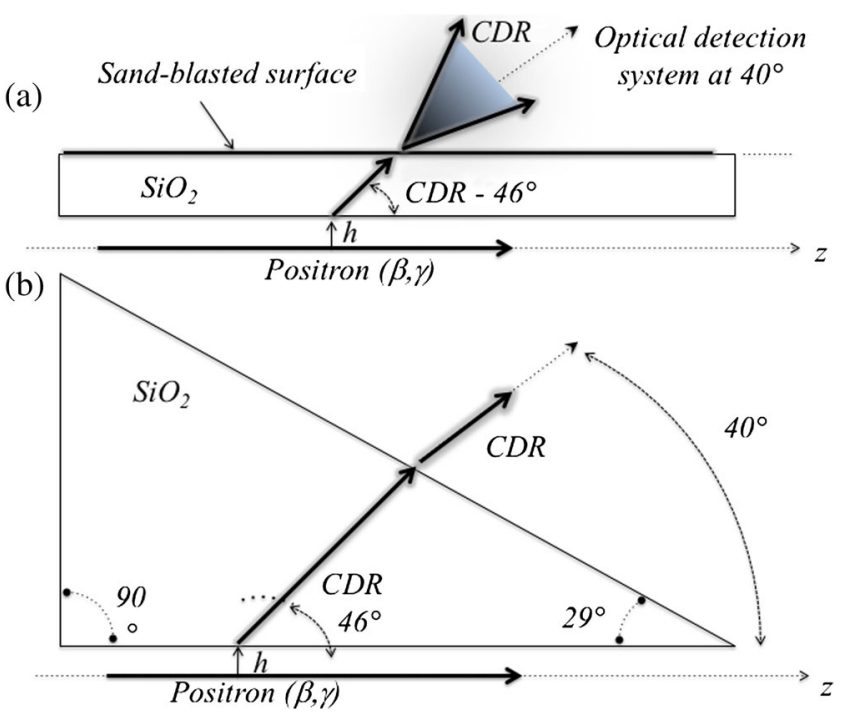

FIG. 1. Emission of Cherenkov diffraction radiation in a flat radiator with a sandblasted outer surface (a) and in a triangular radiator (b). The relative dielectric permittivity of $\mathrm{SiO}_{2}$ is $\varepsilon[0.2-2 \mu \mathrm{m}] \approx 2.1 \varepsilon_{0}$.

different design have been constructed and mounted onto a mechanism with 2 degrees of freedom: a translation to insert the radiator once the beam is circulating in the ring and a rotation to allow a precise steering of the emitted photons through the optical detection line. The first target, made out of Corning high purity fused silica $\left(\mathrm{SiO}_{2}\right) 7980$ and shown in Fig. 1(a), has a rectangular shape. The outer surface of the target has been sandblasted such that the CDR emitted inside the target at an angle of $46^{\circ}$ with respect to the beam trajectory is scattered out. A small fraction of the photons will then be captured by our optical system, which is, by design, detecting photons, emitted at an angle of $40^{\circ}$ relative to the incoming positron beam trajectory. The second target, built using the same material, has a triangular shape as depicted in Fig. 1(b). The outer face of the radiator was designed with an angle of $29^{\circ}$ such that the Cherenkov photons are refracted towards the detection system. This design would preserve the angular distributions of CDR and allow accurate measurements of its polarization content. The angular acceptance of the optical detection line is limited to $20 \mathrm{mrad}$ by design, which means that the amount of light detected from the flat depolished radiator will be smaller (i.e.,1/6000) than the one from the prismatic target due to the diffusivity of the outer surface of the flat target.

The equation for the PR spectral-angular distribution for such geometries is presented by Eq. (18) in [24]. Using this formula and the beam parameters of CESR, the photon spectra emitted by a single particle have been calculated and the results are presented in Fig. 2 for different impact parameters. In the model, the dielectric properties of the target are defined from the three-term Sellmeier dispersion equation [28]. As shown in Fig. 2, for larger impact 


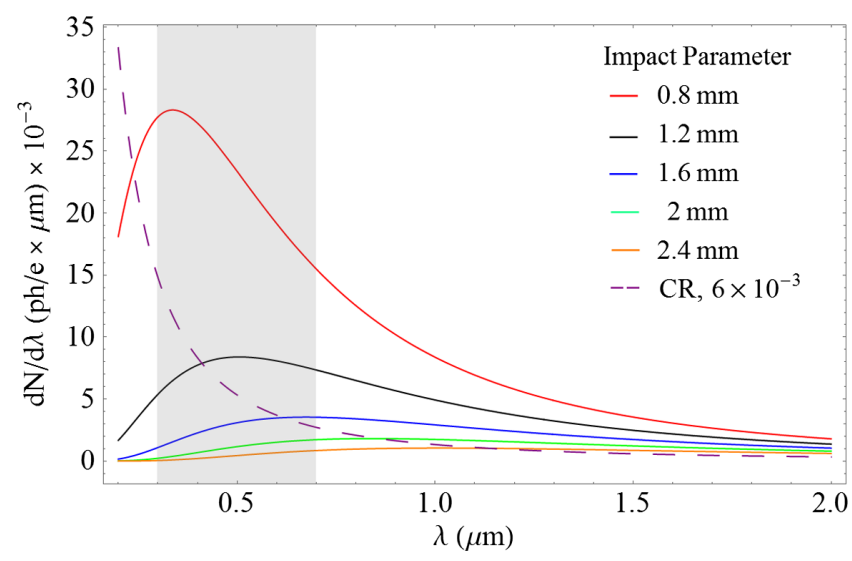

FIG. 2. Cherenkov diffraction photon spectrum from a $5.3 \mathrm{GeV}$ positron propagating at impact parameters of $0.8,1.2,1.6,2$, and $2.4 \mathrm{~mm}$. The gray area represents the spectral sensitivity of the camera. The dashed curve represents the Cherenkov radiation (CR) spectrum emitted by a particle traveling inside the dielectric and has been scaled down by $6.5 \times 10^{-3}$ for better presentation.

parameters, the CDR intensity decreases significantly. The light spectrum for shorter wavelengths falls off due to the attenuation of the effective radius of particle electromagnetic field. The light intensity emitted at longer wavelengths is reduced following the well-known Cherenkov spectral dependency [2].

The detection system relies on an intensified camera with a multiple-alkali-metal photocathode providing a high quantum efficiency in the wavelength range 300-700 nm, highlighted in Fig. 2 by the gray area. The optical system is equipped with a polarizer and a $10 \mathrm{~nm}$ bandpass optical filter centered at $600 \mathrm{~nm}$. Two lenses, mounted on flippers, can be inserted either to image the radiator using a $150 \mathrm{~mm}$ focal length achromatic lens or to measure the angular distribution of the radiation with a $500 \mathrm{~mm}$ focal length plano-convex lens. Experimentally, the best signal to noise ratio on the camera is achieved by adjusting both the gain of the image intensifier and the integration time of the camera.

Three-dimensional models of the interaction using the flat and prismatic radiators are depicted in Figs. 3(a) and 3(b), respectively. For clarity, on the right side of Fig. 3, the raw images are presented with the projection of the radiators on the camera plane. The beam footprint is clearly visible along
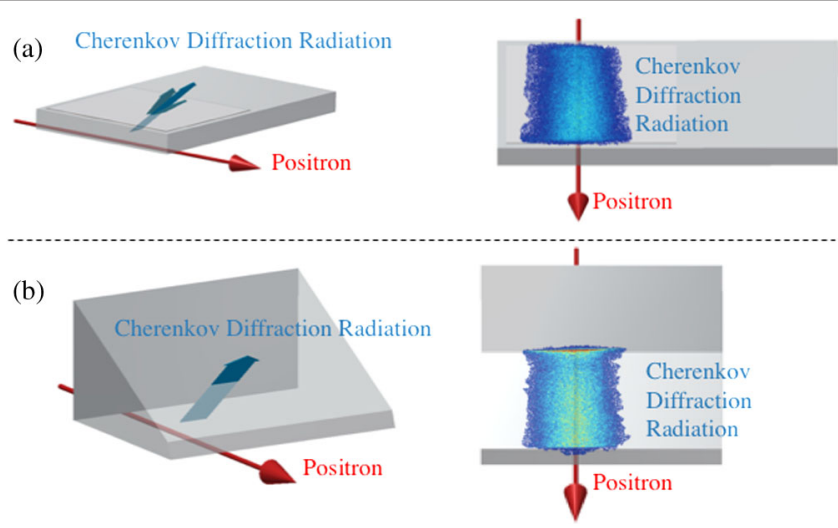

FIG. 3. 3D model of the interaction and imaging the beam using the flat target (a) or with the prismatic target (b).

the length of the dielectric as the beam propagates underneath it. From the raw images, a constant background level is estimated and subtracted from the whole image.

A set of images from the flat Cherenkov radiator, acquired with a $300 \mathrm{~ms}$ camera integration time and maximum gain on the image intensifier, are presented in Fig. 4 for different beam impact parameters ranging from 0.9 to $1.37 \mathrm{~mm}$. Experimentally, this was achieved by moving the beam vertically closer to the surface of the radiator. The measured light intensity increases significantly for smaller impact parameters. One can note that the amount of light measured along the radiator is not constant as visible in Fig. 4(e). This effect is believed to be due to a tilt angle (i.e., $1.9^{\circ}$ ) between the vertical beam trajectory and the surface of the radiator. In this condition, the light produced at the beginning and the end of the dielectric will change according to the distance between the particle and the surface of the material. We also studied if this reduction in light intensity could come from vignetting effects due to the limited angular aperture of the optical system, but the diffusivity of the sandblasted surface is so large that we expect the light to be scattered up to a very broad angle, such that vignetting effects can be discarded. In Fig. 4, one can also note that the beam trajectory is tilted in the horizontal plane as represented by the red line overlaid on each image. This would indicate a horizontal tilt angle of the beam of $3.7^{\circ}$ with respect to the axis of the radiator. This demonstrated the possibility of measuring both
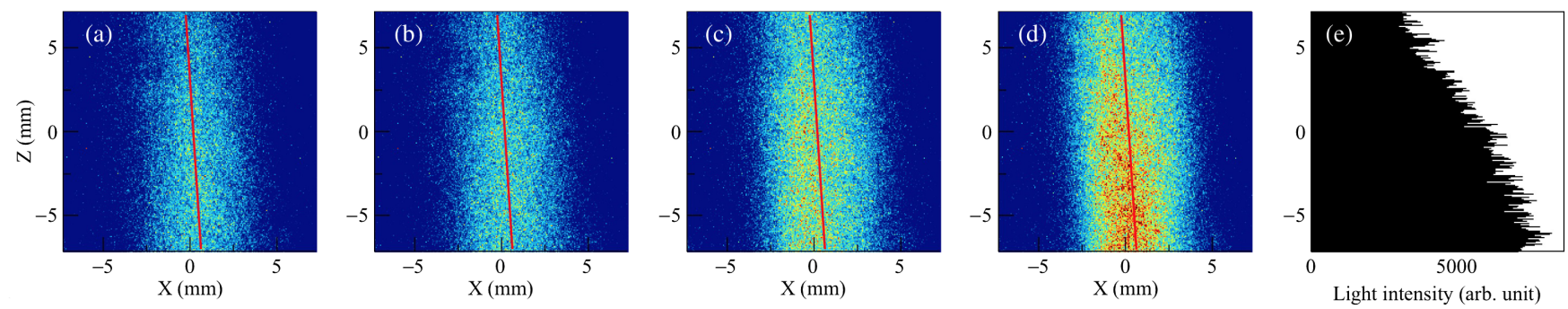

FIG. 4. Images acquired using the flat target, with no polarizer and a $600 \pm 10 \mathrm{~nm}$ bandpass filter, for different impact parameters: (a) 1.37 , (b) 1.32 , (c) 1.08 , (d) $0.9 \mathrm{~mm}$. (e) Projection of the image (d) along the $z$ axis. 


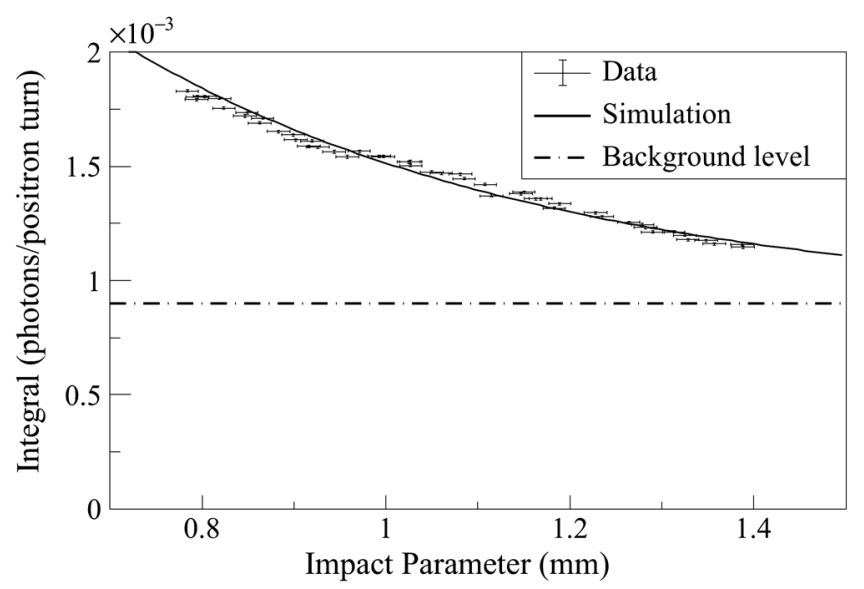

FIG. 5. Experimental and simulated CDR light intensity emitted as function of impact parameter at $600 \pm 10 \mathrm{~nm}$.

the horizontal and vertical tilt angles of the beam with respect to the radiator.

The total photon intensity emitted by CDR is calculated by summing up the amplitudes from all of the pixels in the acquired images. Its evolution as a function of the impact parameter is presented in Fig. 5 with theoretical predictions. Experimentally, reliable data could not be acquired for impact parameters smaller than $0.8 \mathrm{~mm}$. Below this value, in fact, the beam lifetime is strongly reduced due to the scraping of beam tails as they directly impact on the radiator. The data points are plotted in photon per particle per turn, taking into account the measured beam current. As expected from polarization radiation theory, the light intensity increases exponentially for smaller impact parameters. The background level is indicated by the dashed line in Fig. 5. Using calibrations performed with a laser source, the light intensity produced at a wavelength of $600 \pm 10 \mathrm{~nm}$ in the $2 \mathrm{~cm}$ long prismatic radiator corresponds to $0.8 \times 10^{-3}$ photon per turn per particle for an impact parameter of $1 \mathrm{~mm}$, which is in good agreement with our predictions. This would bring the photon intensity radiated in the entire wavelength range of 200-2000 $\mathrm{nm}$ at 0.1 photon per turn per particle for a $1 \mathrm{~mm}$ impact parameter. This number is comparable to the light intensity generated by backward transition radiation $[29,30]$ and exceeds, by at least one order of magnitude, the light intensity emitted by backward diffraction radiation from a conducting slit of similar aperture [20,21]. This result contradicts the discussion of Ginzburg reported in [31] assuming that the Cherenkov radiation can only be generated at impact parameters comparable to or smaller than the radiation wavelength.

Using the prismatic radiator, a systematic measurement of the angular distribution of CDR was performed for different polarizations. The angular distributions measured, using a $1.2 \mathrm{~ms}$ camera integration time and a low gain on the image intensifier (i.e., 50 times smaller than one of images presented in Fig. 4), for horizontally and vertically polarized photons are depicted in Figs. 6(a) and 6(b),
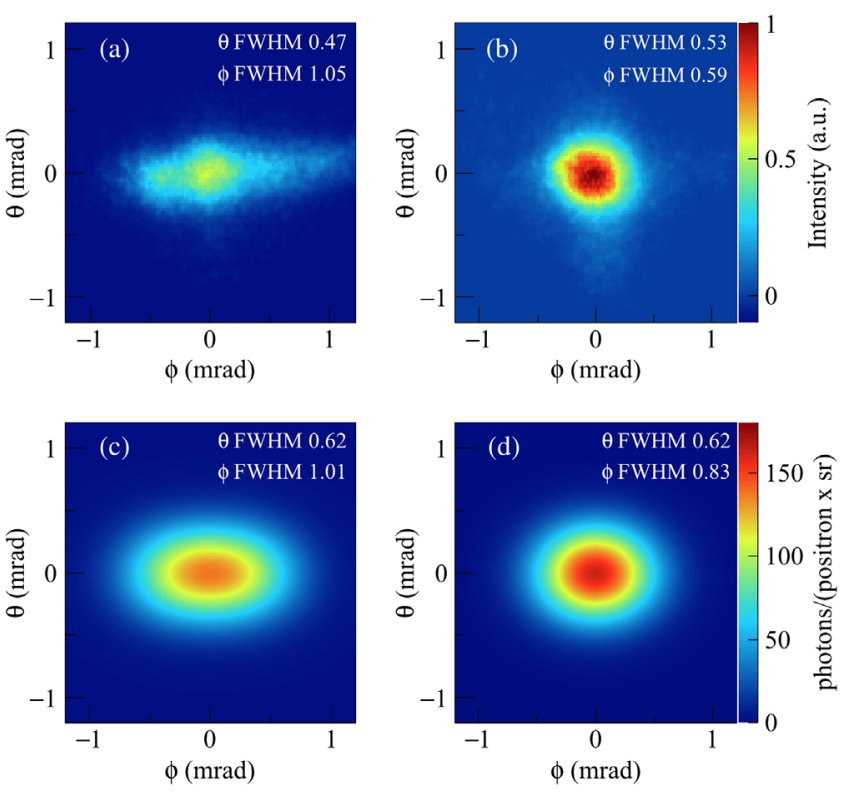

FIG. 6. Comparison between the simulated and measured angular distributions of CDR for (a),(c) horizontally and (b), (d) vertically polarized photons. Measurements were performed for a wavelength of $600 \pm 10 \mathrm{~nm}$.

respectively. The simulated angular distributions, shown in Figs. 6(c) and 6(d), were calculated according to Eq. (18) from [24] and take into account the horizontal and vertical beam divergences of 270 and $18 \mu \mathrm{rad}$, respectively, as extrapolated from emittances measured using synchrotron light monitors [32]. The experimental data are consistent within $30 \%$ with simulations showing that the radiation is confined within submilliradian cone angles, both polar $(\theta)$ and azimuthal $(\phi)$ angles, in contrast to classical Cherenkov radiation, for which the angular distributions have a conical shape centered at the Cherenkov angle.

Beam images were also acquired using the prismatic radiator and are presented in Fig. 7. The horizontal beam profiles obtained using horizontally polarized photons presents unrealistic large tails, whereas the same measurement performed with vertically polarized photons has a Gaussian shape with a full width half maximum value of $4 \mathrm{~mm}$, agreeing within $10 \%$ with the horizontal beam size measured with the synchrotron light interferometer [32].

In conclusion, we have performed the first observation and systematic study of incoherent Cherenkov diffraction radiation emitted by a $5.3 \mathrm{GeV}$ positron in a $2 \mathrm{~cm}$ long fused silica radiator. The experimental results highlight the similitudes and differences between CDR and classical Cherenkov radiation emitted when a particle travels through matter. As the photons are emitted in a narrow cone centered at a large angle, CDR will provide a great opportunity to develop high-quality and simple noninvasive beam diagnostics for high-energy charged particle beams. The exponential decay of the light emitted for larger impact parameters is a signature of CDR and can provide very 

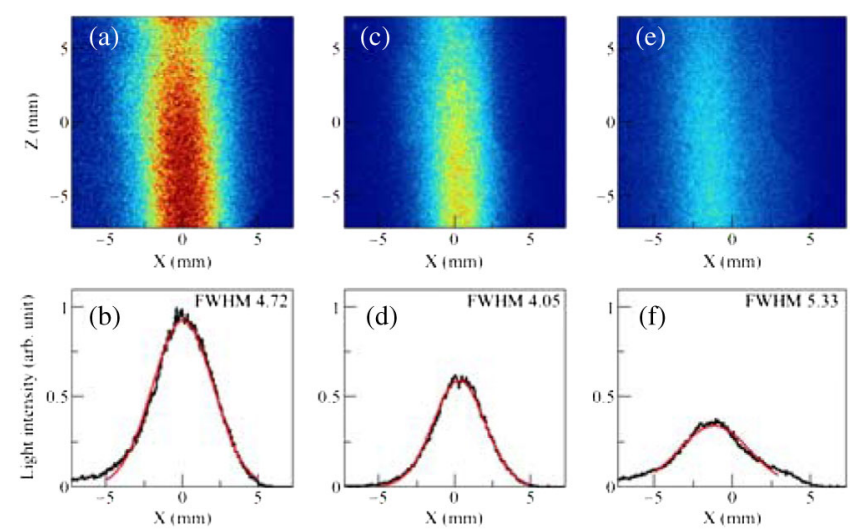

FIG. 7. Beam images and corresponding horizontal beam profiles acquired with the prismatic radiator with no polarizer (a),(b), with a vertical (c),(d) and a horizontal (e),(f) polarizer.

sensitive measurements of the beam position, surpassing what has been achieved using optical diffraction radiation [26]. This will find applications for centering beams in dielectric capillaries, which are typically used in DWA $[19,20]$, plasma lenses [33], or in bent-crystal collimators [34]. Two different radiator designs were tested successfully and allowed precise measurement of the angular distribution of the radiation as well as its light intensity, proven to be in good agreement with our theoretical model. The horizontal size of the Cornell positron beam was also measured using CDR and was found to be in good agreement with other detection techniques. Future experimental and theoretical investigations will study the resolution limit of CDR for transverse beam size and beam tilt angle measurements.

The authors are thankful for the Tomsk Polytechnic University Competitiveness Program grant, the Ministry of Education and Science of the Russian Federation (Projects No. 0.1757.GZB.2017 and No. 3.1903.2017/4.6, program "Nauka").

*robert.kieffer@cern.ch

[1] P. A. Cherenkov, Visible radiation produced by electrons moving in a medium with velocities exceeding that of light, Phys. Rev. 52, 378 (1937).

[2] I. E. Tamm and I. M. Frank, Coherent radiation of a fast electron in a medium, Sov. Phys. Dokl. 14, 107 (1937).

[3] I.E. Tamm, Radiation emitted by uniformly moving particles, J. Phys. (Moscow) 1, 439 (1939).

[4] V.L. Ginzburg, On quantum theory of radiation of an electron uniformly moving in a medium, Sov. Phys. JETP 10, 589 (1940).

[5] T. Kajita, Nobel lecture: Discovery of atmospheric neutrino oscillations, Rev. Mod. Phys. 88, 030501 (2016).

[6] M. Demarteau, R. Lipton, H. Nicholson, and I. Shipsey, Particle and nuclear physics instrumentation and its broad connections, Rev. Mod. Phys. 88, 045007 (2016).
[7] C. W. Fabjan and F. Gianotti, Calorimetry for particle physics, Rev. Mod. Phys. 75, 1243 (2003).

[8] M. Csatari-Divall et al., Fast phase switching within the bunch train of the PHIN photo-injector at CERN using fiberoptic modulators on the drive laser, Nucl. Instrum. Methods Phys. Res., Sect. A 659, 1 (2011).

[9] V. L. Ginzburg and I. M. Frank, Radiation of electrons and atoms moving along the axis of a tube in a dense medium, Sov. Phys. Dokl. 56, 699 (1947).

[10] J. G. Linhart, Cherenkov radiation of electrons moving parallel to a dielectric boundary, J. Appl. Phys. 26, 527 (1955).

[11] R. Ulrich, On the Cherenkov radiation of an electron moving parallel to a dielectric boundary, Z. Phys. 194, 180 (1966).

[12] M. Danos, Cherenkov radiation from extended electron beams, J. Appl. Phys. 26, 2 (1955).

[13] Y. Kalkal and V. Kumar, Analysis of Cherenkov free-electron lasers, Phys. Rev. ST Accel. Beams 18, 030707 (2015).

[14] J.E. Walsh, T. C. Marshall, and S.P. Schlesinger, Generation of coherent Cherenkov radiation with an intense relativistic electron beam, Phys. Fluids 20, 709 (1977).

[15] W. Main, R. Cheery, and E. Garate, 200 MW S-band dielectric Cherenkov maser oscillator, Appl. Phys. Lett. 55, 1498 (1989).

[16] W. Peter, E. Garate, W. Main, and A. Fisher, High-Gain X-Band Dielectric Cherenkov Maser, Phys. Rev. Lett. 65, 2989 (1990).

[17] F. Ciocci, A. Doria, G. P. Gallerano, I. Giabbai, M. F. Kimmitt, G. Messina, A. Renieri, and J.E. Walsh, Observation of Coherent Millimeter and Submillimeter Emission from a Microtron-Driven Cherenkov Free Electron Laser, Phys. Rev. Lett. 66, 699 (1991).

[18] J. Ohkuma, S. Okuda, and K. Tsumori, Measurement of Coherent Cherenkov Radiation from an Intense Beam of a Picosecond Electron Bunch, Phys. Rev. Lett. 66, 1967 (1991).

[19] W. Gai, P. Schoessow, B. Cole, R. Konecny, J. Norem, J. Rosenzweig, and J. Simpson, Experimental Demonstration of Wake-Field Effects in Dielectric Structures, Phys. Rev. Lett. 61, 2756 (1988).

[20] B. D. O'Shea et al., Observation of acceleration and deceleration in gigaelectron-volt-per-metre gradient dielectric wake-field accelerators, Nat. Commun. 7, 12763 (2016).

[21] A. P. Potylitsyn et al., Diffraction Radiation from Charged Particles, STMP 29 (Springer Berlin, 2010).

[22] D. V. Karlovets and A.P. Potylitsyn, Diffraction radiation from a finite-conductivity screen, J. Exp. Theor. Phys. 90, 326 (2009).

[23] M. V. Shevelev, A. Konkov, and A. Aryshev, Soft x-ray Cherenkov radiation generated by a charged particle moving near a finite-size screen, Phys. Rev. A 92, 053851 (2015).

[24] M. V. Shevelev and A. S. Konkov, Peculiarities of the generation of Vavilov-Cherenkov radiation induced by a charged particle moving past a dielectric target, J. Exp. Theor. Phys. 118, 501 (2014).

[25] P. Karataev, S. Araki, R. Hamatsu, H. Hayano, T. Muto, G. Naumenko, A. Potylitsyn, N. Terunuma, and J. Urakawa, Beam-Size Measurement with Optical Diffraction Radiation at KEK Accelerator Test Facility, Phys. Rev. Lett. 93, 244802 (2004). 
[26] R. Kieffer et al., Optical diffraction radiation for beam position monitoring of charged particle beams, Nucl. Instrum. Methods Phys. Res., Sect. B 402, 88 (2017).

[27] M. G. Billing, The conversion of CESR to operate as the test accelerator, CesrTA. Part 1: Overview, J. Instrum. 10, P07012 (2015).

[28] E. D. Palik, Handbook of Optical Constants of Solids (Academic Press, San Diego, 1998).

[29] V. L. Ginzburg and I. M. Frank, Radiation from a uniformly moving electron passing from one medium to another, Sov. Phys. JETP 16, 15 (1946).

[30] D. Rule, Transition radiation diagnostics for intense charged particle beams, Nucl. Instrum. Methods Phys. Res., Sect. B 24-25, 901 (1987).
[31] V. L. Ginzburg, Radiation by uniformly moving sources (Vavilov-Cherenkov effect, transition radiation and other phenomena), Phys. Usp. 39, 973 (1996).

[32] S. T. Wang, D. L. Rubin, J. Conway, M. Palmer, D. Hartill, R. Campbell, and R. Holtzapple, Visible-light beam size monitors using synchrotron radiation at CESR, Nucl. Instrum. Methods Phys. Res., Sect. A 703, 80 (2013).

[33] J. Van Tilborg et al., Active Plasma Lensing for Relativistic Laser-Plasma-Accelerated Electron Beams, Phys. Rev. Lett. 115, 184802 (2015).

[34] W. Scandale et al., Comprehensive study of beam focusing by crystal devices, Phys. Rev. Accel. Beams 21, 014702 (2018). 